\title{
High Dynamical Range Observations on the VLT
}

\author{
Anne M. Lagrange \\ Laboratoire d'Astrophysique de Grenoble; Université J. Fourier, BP 53; \\ F38041 Grenoble Cedex \\ Gael Chauvin, David Mouillet, Jean-Luc Beuzit and the NAOS \\ Consortium \\ Laboratoire d'Astrophysique de Grenoble; Université J. Fourier, BP 53; \\ F38041 Grenoble Cedex \\ ONERA
}

\begin{abstract}
The recently installed Adaptive Optics system NAOS now offers diffraction limited images at the VLT. Together with the CONICA camera, NAOS provides the possibility to perform high dynamical range observations in the near IR domain. We present here NAOS-CONICA (hereafter NACO) capabilities as well as the first images of binaries obtained during NACO commissioning.
\end{abstract}

\section{Introduction}

One of the today astrophysical challenges is the detection and characterization of faint companions, low mass stars, brown dwarfs (BDs) and exo-planets (EPs), close to bright stars. Star, BD and EP formation scenarios indeed require statistical observational constrains (mass ratio, separations) which are sofar mostly lacking at short separations and/or high contrasts.

The detection of faint companions close to bright objects requires excellent image quality hence from the ground, the use of adaptive optics (AO). They also require excellent detector performances and dedicated devices such as coronagraphs, dual imagers or polarimeters. Since the first coupling of coronagraph with AO at ESO (Beuzit et al. 1996; Lagrange et al. 2001) several telescopes have been equipped with such devices, and many results have been obtained in this field, concerning mostly (very) low mass stars and to a lower extent, BDs, as seen in these proceedings. Imaging of exoplanets has remained out of the scope of previous instruments but in some cases can be tackled nowadays. Dual imaging (Marois et al. 2000) and dual polarimetry (e.g. Kuhn et al. 2001; Potter et al. 2002) potentially allow for a significantly increased detection limit. The spectral characterization of very late type stars and BDs has also been the subject of intensive work recently (see these proceedings) as photometric characterisation gives only rather limited constrains. Again, coupling spectroscopy with $\mathrm{AO}$ allows to characterize faint companions close to bright objects (see e.g. Goto et al. 2002). 

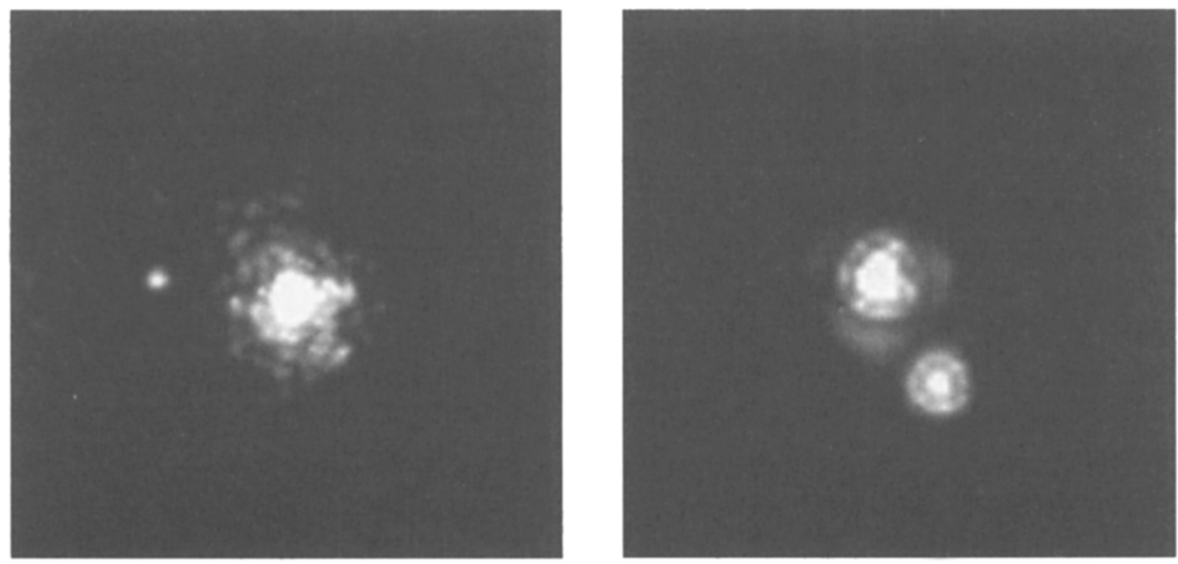

Figure 1. NACO images of binary stars. Left: $\Delta \mathrm{K}=4.2$ at 0.5 ". Central star has a $\mathrm{V}=10$ magnitude. Right: $\Delta \mathrm{K}=1.1$ at 0.2 " around a 8th magnitude star.

\section{NAOS CONICA description}

The NAOS system (Rousset et al. 2000) on the VLT has been designed to provide diffraction limit images with high Strehl ratio in the near IR (1-5 $\mu \mathrm{m}$ domain). NAOS is equipped with a Shack Hartman analyser and a 185 actuators deformable mirror. One sofar unique and useful feature is the possibility to use either a visible wavefront sensor or an IR one, the latter being particularly interesting when using red reference objects. On sky performances were measured during NAOS commissioning during the past six months (Brander et al. 2002; Mouillet et al. 2002). Under medium seeing $\left(0.6-0.8^{\prime}\right)$, NAOS provided Strehls up to $50-55 \%$ at $\mathrm{K}$ band on bright objects. These values included operational limitations (telescope vibrations, remaining static aberrations, non stationary turbulence) on top of the loop itself performance ( $~ 70 \%$ under Kolmogorov conditions). They can therefore be improved in the future.

NAOS feeds an infrared (1-5 $\mu \mathrm{m})$ camera (Lenzen et al. 2000) equipped with an InSb $1 \mathrm{~K} \times 1 \mathrm{~K}$ detector. It offers various observing modes, among which classical imaging in broad and narrow bands, Lyot coronagraphy with occulting masks $0 . " 7$ and $1 . " 4$ in diameter plus Lyot stop at pupil plane, long slit (27!') spectroscopy $(\mathrm{R}=500-1000)$, Fabry Perot and polarimetry, in particular dual Wollaston.

\section{Faint companions detection with NACO}

\subsection{Observations}

NAOS and CONICA have been installed and got first light in November 2001 on UT4 at VLT. In the course of NACO commissioning, a limited number of observations (images and spectra) of close binaries, sometimes with high contrasts were performed. Even though these observations were performed while NACO 

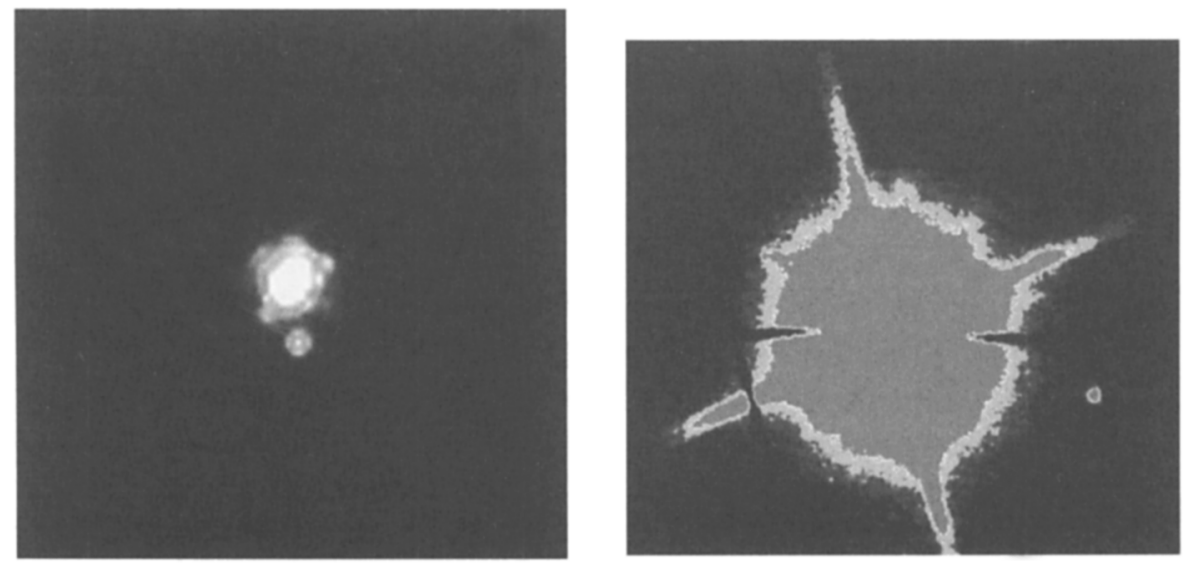

Figure 2. Coronagraphic image of a binary system. Left: $\Delta \mathrm{K}=3$ at 0.3 " around a 9.4th magnitude star. Right (coronagraphy): $\Delta \mathrm{K}=$ 10 at 4.5 " around a 6 th magnitude star. Mask size $=0.35$ " in diameter.

was still under tuning, they are very encouraging. Figures 1 and 2 give examples of images of binary systems, without and with a coronagraph. Obviously, high contrasts can be reached.

\subsection{Simulations of BDs and EP imaging}

Figure 3 shows the expected performances in terms of detection limits of faint objects close to bright ones. The simulations assume a medium seeing $\left(0 .{ }^{\prime \prime} 65\right)$ and a Strehl ratio of $47 \%$. The classical sources of noise (detector, background, photon and speckle) are taken into account. Expected contrasts of giant EPs around young stars in close-by associations such as TW Hydrae, based on the DUSTY model (Chabrier et al. 2000), are overplotted. This example shows that detections of BDs or still hot giant planets around young stars in close-by associations such as TW Hydrae are possible. Also, detections of BDs around long term RV variable stars are feasible; this will allow to significantly increase the overlap domain in Mass-Period diagram between both types of searches.

\section{References}

\section{References}

Beuzit, J.L., Mouillet, D., Lagrange, A.M., et al 1997, A\&AS, 125, 175

Beuzit, J.L., Ségransan, D., Forveille, T. 2001, A\&A, submitted

Bocalletti A., Moutou, C., Mouillet, D., et al 2001, A\&A, 367, 37

Brandner, W. et al. ESO Messenger, 2002

Chabrier, G., Baraffe, I., Allard, F., et al 2000 , ApJ, 542, 464

Delfosse X., Forveille, T., beuzit, J.L. 1999, A\&A, 344, 897

Duchêne G., Bouvier, J. and Simon, T. 1999, A\&A, 343, 831 


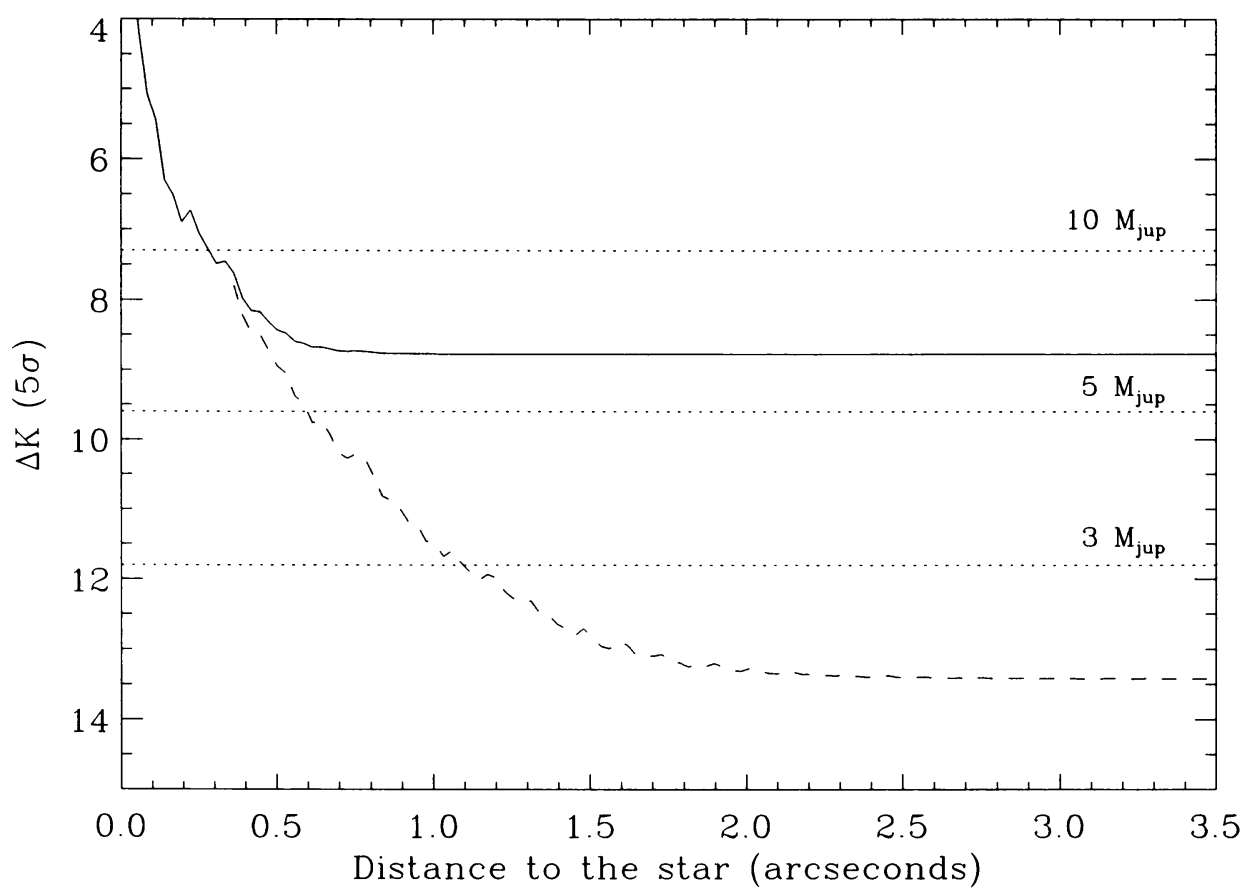

Figure 3. Contrast achievable with NACO for a bright $(\mathrm{K}=7.5)$ star located at TW Hya distance $(\sim 65 \mathrm{pc})$. Solid line: without coronagraph in $\mathrm{Br} \gamma$ narrow band to not saturate the detector. dashed line: with coronagraph in K-band (mask of 0." 7 in diameter). Expected contrasts from young $(\simeq 10 \mathrm{Myr})$ giant EPs with various masses are overplotted (data from Chabrier et al. 2000). 
Els S.G., Sterzik, M.F., Marchis, F., et al 2001, A\&A, 370, L1

Goto, M., Kobayashi, N., Terada, H. et al. 2002, ApJ, 567, 59

Kuhn, J.R., Potter, D., and Parise, B., 2001, 2001, ApJ, 553, L189

Lagrange A.-M. and Mouillet, D. 2000, in From Extrasolar Planets to Cosmology: The VLT Opening Symposium,eds J. Bergeron and A. Renzini, SpringerVerlag, 521

Lenzen R., Hofmann, R., Bizenberger, P. 1998, SPIE, 3354, 60

Marois, C., Doyon, R., Racine, R., Nadeau, D., 2000, SPIE, 4008, 788

Martín, E. L., Brandner, W., Bouvier, J., et al 2000, ApJ, 543,299

Mouillet, D., Lagrange, A.-M., Beuzit, J.-L 1997, A\&A, 324, 108

Mouillet, D. et al. ESO Messenger, 2002, in press

Potter, D., Martín, E. L., Cushing, M. et al. 2002, ApJ, 567, L133

Rigaut F., Salmon D., Arsenault R., et al 1998, PASP, 110, 152

Rousset G., Beuzit J.-L. 1999, in Adaptive Optics in Astronomy, Cambridge University Press, F. Roddier Ed., 171

Rousset, G., Lacombe, F., Puget, P. 2000, SPIE, 4007, 72

Ségransan, D., Delfosse, X., Forveille, T. 2000, A\&A, 364, 665 


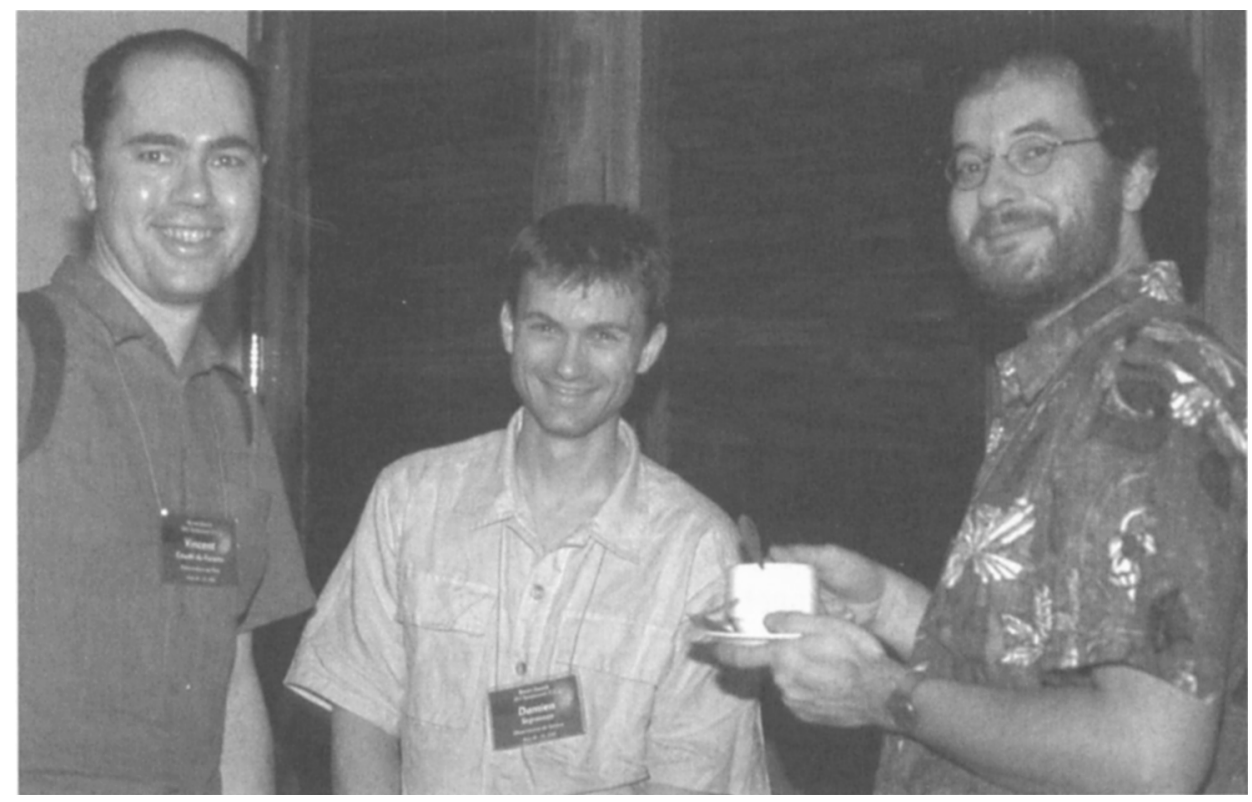

From left to right: Vincent Coudé du Foresto, Damien Segransán, and Jean-Luc Beuzit during a coffee break 